\title{
FAST 3D SEGMENTATION OF HEPATIC IMAGES COMBINING REGION AND BOUNDARY CRITERIA
}

\author{
Daniel Reska, Cezary Boldak, Marek Kretowski
}

Faculty of Computer Science, Bialystok University of Technology, Bialystok, Poland, d.reska@pb.edu.pl

\begin{abstract}
A new approach to the liver segmentation from 3D images is presented and compared to the existing methods in terms of quality and speed of segmentation. The proposed technique is based on 3D deformable model (active surface) combining boundary and region information. The segmentation quality is comparable to the existing methods but the proposed technique is significantly faster. The experimental evaluation was performed on clinical datasets (both MRI and CT), representing typical as well as more challenging to segment liver shapes.
\end{abstract}

\section{Introduction}

Development of effective and reliable segmentation methods is one of the most important tasks in the medical image analysis. Outlining of the organs and pathological changes is the necessary stage in the preoperative planning [12], diagnostics and visualization. The manual segmentation is labor-intensive, especially in the case of large MRI or CT data sets. Usage of reliable segmentation tools can shorten time and increase precision and reproducibility of the results.

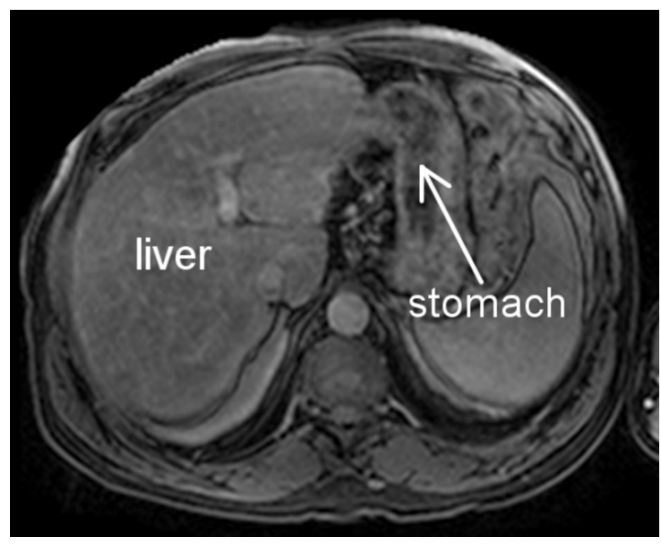

Fig. 1: Problems with the liver segmentation: insufficient separation from other organs and ambiguous boundaries.

The liver segmentation is a particularly difficult task. Irregularity of the liver shape and ambiguous boundaries with the adjacent organs (see Fig. 1) are the greatest challenges for automatic and semi-automatic methods [7].

In this work we propose a new 3D segmentation method based on geometric deformable models [13]. This method uses an active 3D surface that expands inside the organ volume. The process is driven by the edge information and intensity statistics of the surface interior. The proposed method was tested on various hepatic data sets and evaluated with the standard segmentation quality measures. This approach was implemented in our in- 
tegrated segmentation environment and it enabled us to compare it with our previously developed tools.

\section{Segmentation methods}

Several methods for the liver segmentation were used in the past [7]. One can order them using several criteria:

a) interactive (where a trained operator guides the method in some way to get valuable results $[15,2])$ vs. $a u$ tomatic (where very little or no user interaction is needed at all $[22,3])$ : in general the interaction assures better quality but increases the total segmentation time and makes it more tedious;

b) pixel-based (where the segmented object is a simple collection of independent points [16]) and producing higher-level shapes (where it is represented by some ordered structures: lines, surfaces, etc. [6, 22]);

c) model-based (where the method is preliminary trained on manually labeled images to discover some knowledge about the segmented data [22, 6]) vs. freeform (where the method does not need the training stage [15]): the need of training data is sometimes a problem, moreover - only cases similar to the training data sets are well segmented;

d) $2 \mathrm{D}$ (where each $2 \mathrm{D}$ slice is segmented separately, sometimes using an initialization from the previous slice [21], and the volume is reconstructed from the independent 2D segmentations [5]) vs. true $3 D$ (where the complete 3D neighborhood of each segmented point is taken into account $[6,2])$ : in general the true 3D approach yields more coherent results, but can be difficult to construct and implement.

Segmentation of the 3D images is in general a timeconsuming task. Methods evaluated in [7] are reported to take several minutes (except the simplest region growing) to segment one image on a standard computer (from 5.5 to 60 minutes). It makes the interactive work with them (e.g. during a medical consultation) almost impossible.

In our previous work we focused on interactive 2D methods [14]. Currently, the development of the active contour based methods (snakes) are the main focus of our research. The snake is defined as a deformable parametric curve, which deforms under influence of internal and external forces. The goal of the evolution process is to minimize the total energy of the snake. With the contour defined as $v(s)=(x(s), y(s))$ where $s \in[0,1]$, the total snake energy could be written as:

$$
\begin{aligned}
& E_{\text {snake }}^{*}=\int_{0}^{1} E_{\text {snake }}(v(s)) d s \\
& =\int_{0}^{1} E_{\text {int }}(v(s))+E_{\text {image }}(v(s))+E_{\text {con }}(v(s)) d s
\end{aligned}
$$

where $E_{\text {int }}$ is the internal energy (controlling bending and stretching), $E_{\text {image }}$ is the image force (moving the snake toward desired features) and $E_{c o n}$ represents other possible constraints. The original snake model [9] have significant limitations. The necessity of initialization near the segmented object boundaries led to the introduction of inflation forces [4] or local gradient expansion [20] that would push the snake toward desired image features. Contour subdivision methods [10] were also introduced to overcome the inflexibility of the standard snake fixed topology. Many 3D deformable surfaces were also proposed [13] and successfully used in medical image segmentation [19].

\section{Combining region and boundary criteria}

In this work we present a new segmentation method based on Geometrically Deformed Model (GDM) [13]. This model is represented as a deformable polygon mesh that evolves under the influence of external image features and internal smoothness constraints. Normal vectors of the surface are used as a direction of the deformation of each 
vertex, giving the surface the ability to fill the segmented region.

\subsection{Surface evolution}

The evolution process aims to minimize the global energy of the surface by moving the vertices to new positions with a lower potentials. The potential function $C(v)$ at the current location of the vertex $v$ can be described as

$$
C(v)=a_{0} D(v)+a_{1} I(v)+a_{2} T,
$$

where $D(v)$ is the directional deformation potential, $I(v)$ is the image features term, $T$ is the topology smoothness measure and $a_{0}-a_{2}$ are the weights. The image term is originally defined as

$$
I(V)= \begin{cases}0 & \operatorname{Img}(v)<t \\ \operatorname{Img}(v)-t & \operatorname{Img}(v) \geq t .\end{cases}
$$

where $\operatorname{Img}(v)$ is the data intensity at the position of the voxel $v$ and $t$ is the intensity threshold of the segmented object. The smoothness of the mesh $T$ is assured by keeping each vertex as close as possible to its neighbors, maximizing the coplanarity of the model faces.

The model is also capable to dynamically adapt its topology using a subdivision procedure that multiplies the points count in order to increase the accuracy of the segmentation. This procedure can be performed globally or only on the faces that meet the resampling criteria. The use of the local subdivision condition is necessary, because the redundant resampling increases the computation time and does not affect the final results of the segmentation. The area of a face can be used as a resample condition, enabling the mesh to maintain a given level of detail. Additionally, we use a second condition that prevents the subdivision of the faces composed from vertices that are already placed in their minimum energy positions.

The simplicity of this discrete model allows it to be computationally efficient and easy to visualize. Segmented volume is sampled only on the surface and no information of the interior of the surface is used.

\subsection{Internal region potential}

The incorporation of the internal region characteristics expands the effectiveness of the method, originally making use only of the edge information. The interior of the surface is calculated with an efficient polygon mesh voxelization algorithm [17]. The algorithm uses the raycasting to calculate the model volume: since the surface is closed, counting its intersections with the ray casted from a voxel can tell whether the voxel is inside the surface. The optimized algorithm casts one ray along the entire row of voxels and applies a quad tree partitioning of the surface faces in order to minimize the number of the ray-face intersection tests.

With the internal region computed, the default image term (3) can be modified as:

$$
I(v)= \begin{cases}0 & |\operatorname{Img}(v)-\mu|<k \sigma \\ |\operatorname{Img} g(v)-\mu| & |\operatorname{Img}(v)-\mu| \geq k \sigma .\end{cases}
$$

where $\mu$ and $\sigma$ are the intensity mean and standard deviation of the surface region and $k$ is a user-defined constant. Such a solution eliminates the specific intensity threshold and increases the robustness of the method.

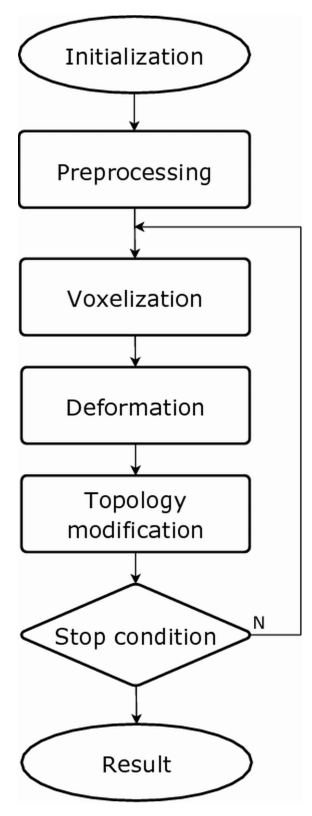

Fig. 2: Block diagram for the proposed algorithm. 


\subsection{Steps of the algorithm}

The proposed segmentation procedure (see Fig. 2) consists of the following steps.

a) Initialization: the user is required to place an initial model inside the liver. The start mesh can be also rescaled to fit the segmented organ. Given the size of the liver, this procedure is not particularly difficult. The start mesh is an icosphere - a spherical solid created by dividing the faces of an icosahedron. The icosphere is used due to its regular topology, suitable for further subdivisions.

b) Preprocessing: in order to smooth the liver internal volume while preserving the edge information, we apply a bilateral filter [18] to the original images (see Fig. 3).

c) Voxelization: the interior of the start surface is calculated and the intensity characteristics (mean and standard deviation) of the region are computed.

d) Deformation stage: the surface vertices are moved to the position of the lowest potential in their neighborhood. The movement direction of each point is calculated from the vectors normal to the faces containing the vertex. The deformation potential pushes the vertex along this vector, resulting in the expansion of the surface. This step continues for a given iteration count, as long as the image and curvature constraints are satisfied. The usual movement distance is constrained to the range of two voxels. The distance can be also reduced by half if the vertex remains unmoved. The movement step and the iteration and reductions count can be adjusted by the user.

e) Topology modification: the surface mesh is refined using the given area and movement criteria. Additionally to the minimal face area condition, an upper limit of the faces number can be set. The final mesh is then returned to the user and another cycle can be performed.

f) Stop condition: the process can stop when no further surface deformation is possible or when the user finds the result satisfying.

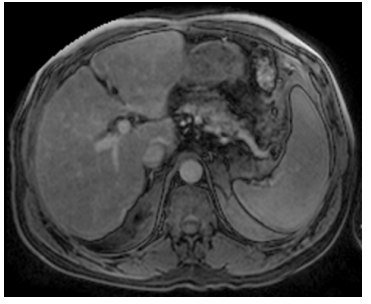

(a)

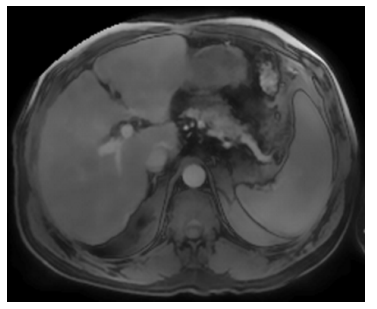

(c)

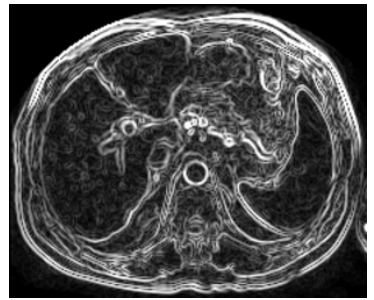

(b)

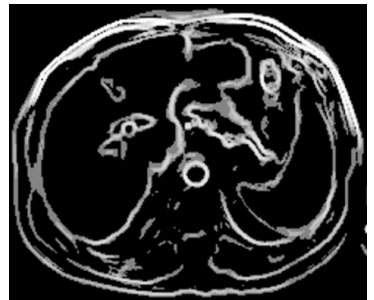

(d)

Fig. 3: The preprocessing step: the edge map (b) generated from the original image (a) contains more undesirable noise than the edge map (d) obtained from the image with the bilateral filter applied (c).

\section{Experimental validation}

The proposed methods were validated on various hepatic MRI and CT sets, gathered at the Pontchaillou University Hospital, Rennes, France. Time and quality of the segmentation were evaluated and compared with other active contour methods using a segmentation testing environment.

\subsection{Framework}

Our method is being developed as a part of an integrated segmentation and visualization environment that provides extensible templates for creating 2D and 3D active contours. Each template can be extended with custom deformation methods, energies/cost functions and other constraints that can be dynamically reconfigured at runtime and extended using a scripting language. The environment is using a server-client architecture, moving the responsibility of computations and data storage from the client to the server. Using the framework we incorporated the standard edge and the proposed region terms into the 
implemented active surface. Then the performance of the proposed approach was compared with our previously developed tools [14]: the region growing and merging with an automatic seed point initialization based on anatomical information and the 2D active contour with an ability to progress over a series of images.

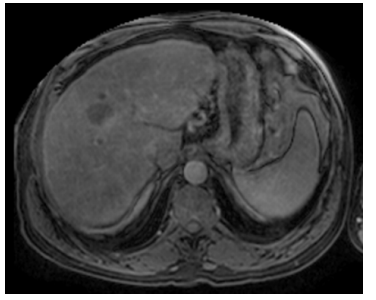

(a)

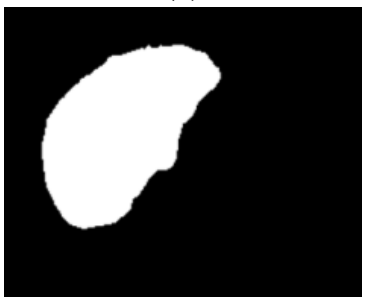

(c)

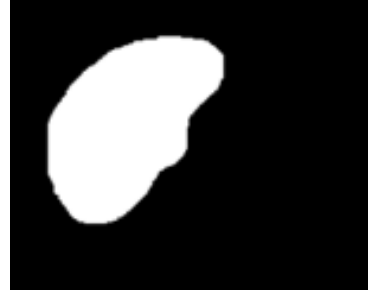

(b)

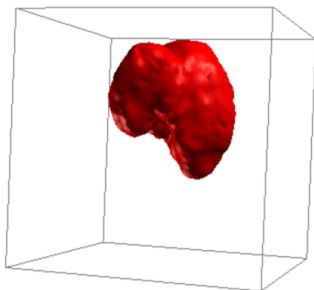

(d)

slice spacing from 2 to $3 \mathrm{~mm}$. We present the method results for three selected data sets:

- MRI-1, containing 100 images of a standard liver MRI;

- MRI-2, containing 100 images of MRI with a pathologically changed liver and particularly separated left and right organ lobes;

- CT-1, with 165 hepatic CT images, selected for evaluating the proposed method on a different imaging modality.

\subsection{Segmentation quality measures}

The segmentation quality was evaluated with three commonly used error measures [7], where $A$ is the tested segmentation and $B$ is the reference. The first one is the Overlap Error (OE), defined as:

$$
O E(A, B)=100(1-(|A \bigcap B| /|A \bigcup B|))
$$

The 0 value indicates that the two sets are identical and 100 that the sets do not overlap.

The second measure is the Relative Volume Difference $(R V D)$, defined as:

$$
R V D(A, B)=100((|A|-|B|) /|B|)
$$

Fig. 4: Segmentation of MRI-1 set: sample image (a) and the manual liver outline (b), corresponding segmentation (c) and the final surface (d).

Combined with other measures that consider the actual set overlap, it can indicate a tendency to over- or undersegmentation of the method.

The third measure is the Average Symmetric Surface Distance (ASD) that computes an average distance (in millimeters) between the surfaces of segmentations $A$ and $B$. With $S(X)$ as the set of surface voxels of segmentation $X$ and $d(v, S(X))$ as a shortest distance from a voxel $v$ to surface $S(X)$, ASD can be defined as:

$$
\begin{gathered}
A S D(A, B)=\frac{1}{|S(A)|+|S(B)|}\left(\sum_{s_{A} \in S(A)}^{d} d\left(s_{A}, S(B)\right)\right. \\
+\sum_{s_{B} \in S(B)} d\left(s_{B}, S(A)\right)
\end{gathered}
$$

The distance of 0 indicates the ideal segmentation.

\subsection{Results}

The manual reference segmentation was performed by an experienced user. The quality measures of the results are presented in Tab. 4.3 and the average time of the segmen- 

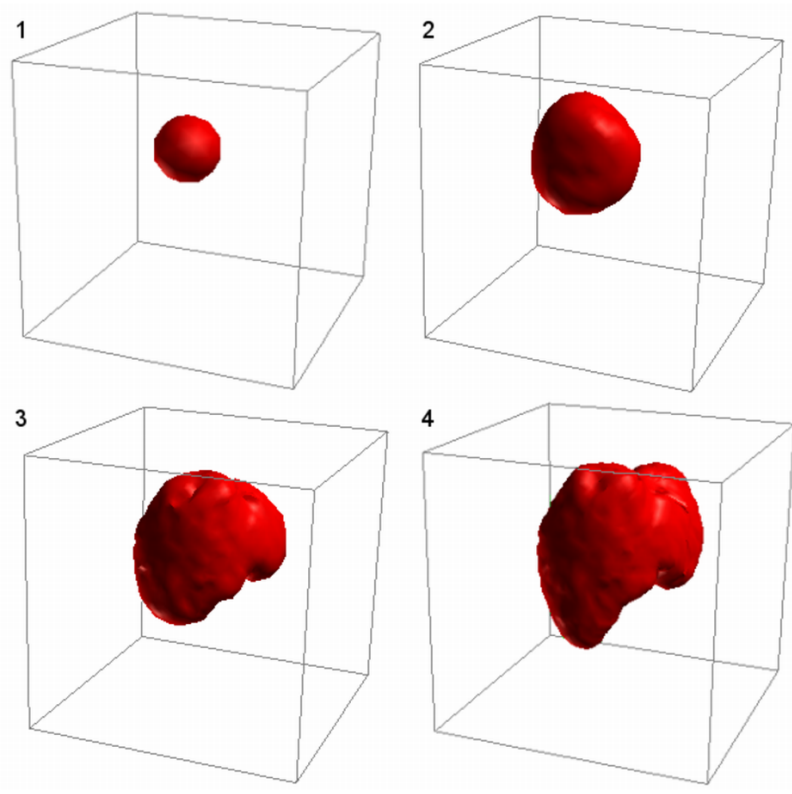

Fig. 5: Surface evolution during four cycles of the segmentation of MRI-1.

\begin{tabular}{|c|c|c|c|c|}
\cline { 3 - 5 } \multicolumn{1}{c|}{} & \multicolumn{3}{c|}{ Measures } \\
\cline { 2 - 5 } \multicolumn{1}{c|}{} & Method & OE & RVD & ASD \\
\hline \multirow{3}{*}{ MRI-1 } & Region Grow. & 14.47 & -5.54 & 2.79 \\
\cline { 2 - 5 } & 2D Snake & 9.31 & -4.43 & 2.43 \\
\cline { 2 - 5 } & 3D Surface & 12.10 & -6.89 & 3.07 \\
\hline \multirow{3}{*}{ MRI-2 } & Region Grow. & 16.44 & -10.17 & 2,07 \\
\cline { 2 - 5 } & 2D Snake & 11.27 & -2.40 & 1.89 \\
\cline { 2 - 5 } & 3D Surface & 25.67 & -24.63 & 4,64 \\
\hline \multirow{3}{*}{ CT-1 } & Region Grow. & 8.89 & -0.57 & 0.94 \\
\cline { 2 - 5 } & 2D Snake & 12.45 & 4.17 & 2.61 \\
\cline { 2 - 5 } & 3D Surface & 14.10 & -7.83 & 1.71 \\
\hline
\end{tabular}

Tab. 1: Segmentation quality

tation is enclosed in Tab. 4.3. The calculations were performed on a desktop machine with $2.66 \mathrm{GHz}$ Core2 Quad processor and 3 GB RAM. The average cycle of the algorithm was $5 \mathrm{~s}$ long and a usual segmentation process required 3 to 5 cycles (see Fig. 5) plus additional 5 seconds for the preprocessing. The usual final surface mesh was composed of approximately 2500 vertices and 5000 triangle faces.

Comparing with our previously developed tools, the proposed active surface method shows a significant time

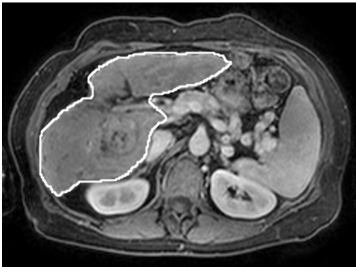

(a)

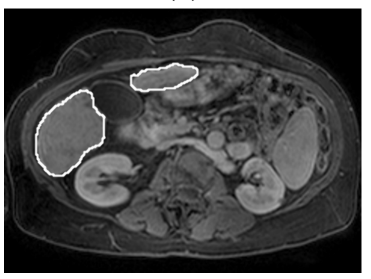

(c)

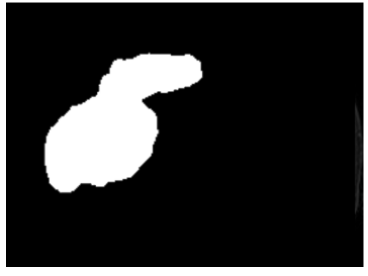

(b)

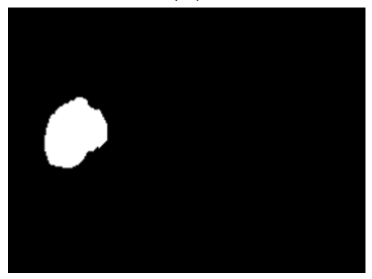

(d)
Fig. 6: Segmentation of MRI-2 set: the final segmentation (b) do not fully match the reference region (a) and one of the liver lobes (c) is completely absent from the segmentation (d).

\begin{tabular}{|c|c|c|c|c|}
\hline Data set & Manual & R.Grow. & Snake & Surface \\
\hline MRI-1 & $25-30$ & $10-15$ & $6-8$ & 0.33 \\
MRI-2 & $40-45$ & $10-15$ & $7-10$ & 0.4 \\
CT-1 & $45-50$ & $28-20$ & $10-15$ & 0.5 \\
\hline
\end{tabular}

Tab. 2: Segmentation time (in minutes)

gain, at the slight expense of the overall quality. The biggest errors could be noticed in the outermost images in the sets, especially at the narrow bottom of the right liver lobe, due to the high force of the curvature constraint and the presence of the partial volume effect [1].

Due to the adaptive nature of the proposed method, the default values of most of its parameters were usually used.

The deformation distance was set to 0.01 (about 2 voxels), with 3 reduction cycles and 10 iterations. These values enabled the fast expansion of the model inside the organ. The curvature level was the parameter that needed some interactive adjustments. To achieve the accurate results near the edges, the curvature had to be slightly decreased in the final cycle of the algorithm.

The volumetric representation of the surface enables the segmentation result to be instantly visualized for further validation and correction. The first set (see Fig. 4) 
represents a fairly standard case, where the results were acceptable without the need of extensive manual refinement. Even with the possible correction, the overall time gain is still preserved. The second set, however, was a greater challenge: the surface usually segmented only one of the main lobes. A precise initialization was necessary to deal with this case, but even with it the results were unsatisfying (see Fig. 6).

\section{Conclusion and future work}

We propose a fast, semi-automatic method for 3D liver segmentation using an active surface that evolves under the influence of edge and region image forces. The method significantly speeds up the segmentation time. However, it shows some difficulties with segmentation of organs with atypical shape and features. The method could be improved with an introduction of a global region of interest [8], which would represent a deformation potential, based on the internal region statistics of the surface. The current intensity-based characteristics could be also replaced with textural features. A more advanced topology resampling algorithm [11] can be used to improve the segmentation of the narrowings in the organ.

The presented method is a part of our currently developed segmentation and visualization environment, that is planned to be made available as a web application. The computational logic of the system will be deployed on powerful multi-core machines. That would open the a possibility of parallelization of the implemented method.

\section{Acknowledgment}

This work was supported with the European funds in the frame of the project "Information Platform TEWI" (Innovative Economy Programme).

\section{References}

[1] M.A. Ballester, A.P. Zisserman, M. Brady, Estimation of the partial volume effect in MRI, Medical Image Analysis, 6(4):389 - 405, 2002

[2] A. Bornik, R. Beichel, and D. Schmalstieg. Interactive editing of segmented volumetric datasets in a hybrid 2-D/3-D virtual environment. In Proc. of ACM Symp. Virtual Reality Software Technol., 197206, 2009

[3] P. Campadelli, E. Casiraghi, and A. Esposito. Liver segmentation from computed tomography scans: A survey and a new algorithm. Artificial Intelligence in Medicine, 45:185-196, 2009

[4] L.D. Cohen. On active contour models and balloons. CVGIP: Image Underst., 53:211-218, 1991

[5] Ch. Florin, N. Paragios, F. Gareth and J. Williams. Liver segmentation using sparse 3D prior models with optimal data support. In Proc. of the 20th Int. Conf. on Information Processing in Medical Imaging, 38-49, 2007

[6] 1. Gao, D. Heath and E. Fishman. Abdominal image segmentation using three-dimensional deformable models. Investigative Radiology, 33(6):348-55, 1998

[7] T. Heimann, B. van Ginneken, M.A. Styner, et al. Comparison and evaluation of methods for liver segmentation from CT datasets. IEEE Trans. on Medical Imaging, 28:1251-1265, 2009

[8] X. Huang, D. Metaxas and T. Chen. Metamorphs: deformable shape and appearance models. IEEE Trans. Pattern Anal. Mach. Intell., 30(8):14441459, 2008

[9] M. Kass, A. Witkin, and D. Terzopoulos. Snakes: 
Active contour models. Int. Journal of Computer Vision, 1(4):321-331, 1988

[10] T. McInerney and D. Terzopoulos. T-snakes: Topology adaptive snakes. Medical Image Analysis, 4(2), 2000

[11] T. McInerney and D. Terzopoulos. Topology adaptive deformable surfaces for medical image volume segmentation. IEEE Trans. on Image Processing, 18(10):840-850, 1999

[12] H.P. Meinzer, M. Thorn, and C.E. Cardenas. Computerized planning of liver surgery - an overview. Computer and Graphics, 26(4):569-576, 2002

[13] J.V. Miller, D.E. Breen, W.E. Lorensen, et al. Geometrically deformed models: a method for extracting closed geometric models form volume data. SIGGRAPH Comput. Graph., 25(4):217-226, 1991

[14] D. Reska and M. Kretowski. HIST - an application for segmentation of hepatic images. Zeszyty Naukowe Politechniki Bialostockiej. Informatyka, 7:71-93, 2011

[15] A. Schenk, G. Prause, and HO. Peitgen.X. Efficient Semiautomatic Segmentation of 3D Objects in Medical Images. In Proc. of Medical Image Computing and Computer-assisted Intervention, 186195,2000

[16] K. Seo, H. Kim, T. Park, P. Kim, and J. Park. Automatic Liver Segmentation of Contrast Enhanced CT Images Based on Histogram Processing. In Proc. of the First Int. Conf. on Advances in Natural Computation - Volume Part I, 1027-1030, 2005

[17] S. Thon, G. Gesquiere and R. Raffin. A low Cost Antialiased Space Filled Voxelization Of Polygonal Objects. GraphiCon 2004, pp. 71-78, 2004
[18] C. Tomasi, R. Manduchi, Bilateral filtering for gray and color images. In In Proc. of the Sixth Int. Conf. on Computer Vision, IEEE Computer Society, 1998

[19] G. Tsechpenakis. Deformable Model-based Medical Image Segmentation. Multi Modality State-ofthe-Art Medical Image Segmentation and Registration Methodologies, Springer Publishing, 2011

[20] C. Xu and J. Prince. Snakes, shapes, and gradient vector flow. IEEE Trans. on Image Processing, 7(3):359-369, 1998.

[21] W.N.J.W. Yussof and H. Burkhardt. 3D Volumetric CT Liver Segmentation Using Hybrid Segmentation Techniques. In Proc. of the 2009 Int. Conf. of Soft Computing and Pattern Recognition, 7(3):404-408, 2009

[22] X. Zhang, J. Tian, K. Deng, Y. Wu, X. Li, Automatic Liver Segmentation Using a Statistical Shape Model With Optimal Surface Detection. IEEE Trans. on Biomedical Engineering, 57(10):26222626, 2010 